\title{
A Network Ridesharing Experiment with Sequential Choice of Transportation Mode
}

\author{
Vincent Mak \\ University of Cambridge \\ Darryl A. Seale \\ University of Nevada, Las Vegas \\ Eyran J. Gisches, Amnon Rapoport \\ University of Arizona \\ Meng Cheng, Myounghee Moon, Rui Yang \\ University of California, Riverside
}

April 10, 2018

Forthcoming in Theory and Decision

Keywords: ridesharing, traffic networks, sequential choice, experiment

Acknowledgement: This research was supported by NSF Grant SES-1418923 awarded to the University of Nevada, Las Vegas and University of Arizona. 


\title{
A Network Ridesharing Experiment with Sequential Choice of Transportation Mode
}

\begin{abstract}
Within the last decade, there has been a dramatic bloom in ridesharing businesses along with the emergence of new enabling technologies. A central issue in ridesharing, which is also important in the general domain of cost-sharing in economics and computer science, is that the sharing of cost implies positive externalities and hence coordination problems for the network users. We investigate these problems experimentally in the present study. In particular, we focus on how sequential observability of transportation mode choices can be a powerful facilitator of coordination in ridesharing. Our study abstracts the essential issues of coordination in ridesharing into a directed network game with experimentally testable predictions. In line with the theoretical analysis, our experimental evidence shows that even a limited extent of sequential choice observability might lead to efficient coordination. However, convergence to efficiency is slower with more limited observability, resulting in a significant increase in travel cost.
\end{abstract}




\section{Introduction}

A fundamental question in economics, transportation science, computer science, and management science concerns the sharing of the cost of joint investment, or the reward for joint effort, in a fair, efficient, and stable way (e.g., Hoefer, 2013; Moulin, 2002; Young, 1994). Most research on cost-sharing in economics has followed two alternative approaches. One approach assumes that the demand and costs are commonly known, and then designs a system of incentives that maximizes some measure of social welfare. The other approach views the cost-sharing problem as a cooperative game, and then proposes game-theoretic solutions such as the core, Shapley value, and nucleolus (Shubik, 1982; Young, 1985, 1988).

In transportation research, the issues of cost allocation have frequently been investigated in the context of ridesharing in traffic networks. With ridesharing, individual travelers share a vehicle (or fleet of vehicles) and split the cost of travel equally (see e.g., Furuhata et al., 2013, for a recent review). The practice of carpooling among commuters is one example. Ridesharing could also describe the economics behind conventional public transportation from shuttle services between suburbs and town centers to buses, trams, and trains. These are all means by which users collectively bear high total transportation costs that become small when a sufficient number of them share the same transportation mode together. Investigations on ridesharing have become especially timely with the recent upsurge in ridesharing businesses such as Uber and Lyft. Essentially the same questions have been studied in computer science for better understanding the recent development of the Internet, in particular e-commerce (Anshelevitch et al., 2008; Syrgkanis, 2010; Jain and Mahdian, 2007).

A central issue in ridesharing, which is also important in the more general domain of costsharing in economics, is that the sharing of cost implies positive externalities and hence coordination problems for the users. The present study is an experimental investigation into these problems. Our choice framework involves a tradeoff that is common among transportation modes with ridesharing characteristics: one transportation mode might be very costly to individual users if a low number of users choose it, but become attractively low-cost to individual users if a sufficiently large number of them choose it; while another transportation mode is less (more) costly than the first one when a low (high) number of users choose it. The problem is to coordinate users to choose the first transportation mode, so that it does operate with sufficiently high usage. A 
simple example is shuttle service versus carpooling between suburbs and town centers: shuttle service might have a higher total operating cost than carpooling, but it might also incur a lower individual cost than carpooling when there is sufficiently high usage. Two of such ridesharing options with tradeoff, together with the baseline option of private car without ridesharing, form a meaningful spectrum of transportation modes for the examination of related tacit coordination problems in our study.

Our work is distinguished from prior literature by our focus on sequential choice observability in ridesharing. Real-time observability of choices of ridesharing transportation modes is often feasible, for example at carpooling junctions on highways. Such observability is nowadays enhanced by online communication platforms and other forms of social media. However, there could still be a limit to which individual users might be informed about all the currently made choices of any transportation mode. As such, it is natural to examine a benchmark case in which every user has complete observability over currently made choices of any transportation mode, and another case in which such observability is only partial. Concern for partial observability in transportation networks is a recently growing development that has generated theoretical research such as Acemoglu et al. (2016). Meanwhile, previous experimental studies on cost-sharing, such as Liu, Mak, and Rapoport (2015) - which employed the choice framework of the present study - are limited to no-information cases without choice observability. Our experimental evidence, driven by theoretical analysis, shows that even a limited extent of sequential choice observability could lead to efficient coordination towards adopting the ridesharing mode. However, in terms of behavioral dynamics, convergence to efficiency would be slower with more limited observability resulting in a significant increase in travel cost.

Our work contributes to two streams of research. First, we contribute to previous experimental research on strategic interactions in transportation mode choices, a relatively nascent area that involves economics, transportation, and operations management research (see Rapoport and Mak, in press, for a recent literature review). Specifically, we extend previous paradigms of decentralized choice of transportation modes in congestible networks (where the externalities that players impose on members of their group are negative) to the choice of transportation modes in directed networks with positive externalities. We also extend previous research on cost-sharing in directed networks from simultaneous (Liu et al., 2015) to sequential transportation mode choices 
with observability, which is arguably more applicable to real-life transportation problems in a world of instant social media communications.

Secondly, we contribute to a considerably larger experimental economics literature on equilibrium selection in coordination games (e.g., Charness et al., 2014; Chen and Chen, 2011; Crawford, 1995; Goeree and Holt, 2005; Van Huyck, Battalio, and Beil, 1990, 1991) that may be traced back to the original contributions on coordination in decentralized systems by Schelling (1960). Most of the research cited immediately above has documented scenarios where tacit coordination fails. In contrast, our present research, as well as previous experimental research on the market entry game (e.g., Erev and Rapoport, 1998; Seale and Rapoport, 2000) and the Braess paradox (e.g., Gisches and Rapoport, 2012; Morgan, Orzen, and Sefton, 2009; Rapoport et al., 2006, 2009, 2014) provides evidence for limited success of tacit coordination in small groups.

\subsection{Research Context}

Ridesharing was introduced in the early $20^{\text {th }}$ century. With mass-produced automobiles flooding the market and shortage of public transportation, car owners in the USA started offering to share their travel for a five-cent streetcar fare. The idea spread from Los Angeles to other parts of the USA, but then declined drastically around 1919 in part due to licensing requirements and liability insurance regulations imposed by local governments. Ridesharing re-emerged in the USA during WWII and subsequently in the 1970s oil crisis with the explicit support and encouragements of local governments, but then its popularity declined sharply with the subsequent fall of the oil prices (see, e.g., Agatz et al., 2012; Berbeglia et al., 2010; Furuhata et al., 2013).

Within the last decade, there has been a dramatic resurgence in for-profit ridesharing operations. These businesses have benefitted from the emergence of new technologies that allow for higher efficiency in communication and greater ability to facilitate the match of supply and demand (e.g., Waze, Google Map). As such, the Nobel Laurette Alvin Roth (http://www.econtalk.org/archives/2015/07/alvin_roth_on_m.html) views Uber as a marketplace that connects drivers and commuters through a private matching algorithm. The modern form of ridesharing (also called "dynamic ridesharing" and "online ridesharing") is a service that is presently offered in cities all over the world. It serves areas that are not covered well, or not covered at all, by public transportation, and often acts as a transit system feeder. It is also capable of serving 
one-time trips, and not only scheduled trips. It attracts commuters mostly because it reduces waiting time, lowers fuel usage, and, most importantly, reduces the transport cost of the commuter.

Research on ridesharing has focused on the coordination of itineraries and travel schedules, on effective methods of encouraging commuters to share travel expenses and reduce pollution, and on the use of new communication capabilities that have been introduced into the market in the last two decades. The need to understand cost-sharing in large unregulated settings such as the Internet or traffic networks has led to recent studies in algorithmic game theory on the design of strategic cost-sharing games to obtain Nash equilibrium properties (Chen, Roughgarden, and Valiant, 2010; Hoefer, 2013).

\subsection{Outline of the Present Setup}

Our study abstracts the essential issues of coordination in ridesharing into a directed network game with experimentally testable predictions. Specifically, we examine cost-sharing in the context of choice of transportation modes in traffic networks with a common origin $(O)$ and common destination $(D)$. The stylized network that we examine has three $(O, D)$ routes corresponding to three transportation modes. One transportation mode (the baseline private car option) has a fixed cost of travel and, therefore, additional commuters choosing this mode impose no externalities. The choice of one of the other two alternative modes of transportation has positive externalities as commuters (players) who choose this mode share its cost of travel: the larger the number of commuters choosing it, the lower the individual cost of travel. As noted previously, we can interpret these two modes as carpool and shuttle.

Another example of a choice framework with a similar structure, which is often observed in airports, is of a scientist attending a conference, who has just completed her flight and has to choose between taking a taxi to the conference center in mid-town and paying a fixed price or, alternatively, joining one of several ridesharing modes of transportation with other participants in the conference who travel to the same destination and agree to divide the cost of travel equally.

A problem that each commuter faces in decentralized networks of this type, where there is no central authority to coordinate transportation mode choices, is as follows. Disregarding other aspects of the transportation mode choice like convenience of travel, search time, etc., in ridesharing transportation, the individual cost of travel is determined by the number of players 
choosing one of the transportation modes. In contrast, the choice of a private mode with a fixed cost of travel is straightforward. The consequences of these choices may be quite different. If relatively few commuters choose ridesharing (and, therefore, each of them incurs a relatively high proportion of the total cost of travel), then the cost of travel of each commuter choosing the private mode may be lower. This inequality is reversed if the number of travelers choosing the same ridesharing transportation exceeds some threshold value which, in turn, depends on the cost structure. Our main purpose in the current paper is to investigate a major topic in tacit coordination regarding how homogenous commuters divide themselves between private transportation and ridesharing modes of transportation. We conduct this investigation under two different variants of the sequential protocol of play, which we describe below.

Protocol of play. In previous experiments on strategic choice of route or transportation mode with either negative (congestion) or positive (cost-sharing) externalities, participants made their choices independently and anonymously under what we call the simultaneous protocol of play (e.g., Gisches and Rapoport, 2012; Liu et al., 2015; Rapoport, Mak and Zwick, 2006; Rapoport, Gisches, and Mak, 2014; Selten et al., 2007). Among these, Liu et al. (2015) examined the same choice framework as in the present study. Those researchers found multiplicity in equilibrium convergence both with their theoretical predictions and experimental data in a setup with the simultaneous protocol. In the present paper, we extend this line of research significantly by investigating the sequential protocol of play, where players choose transportation modes in an exogenously determined order. Under this protocol, which was not examined at all in Liu et al. (2015), transportation mode choices may no longer be considered independent. Instead, the choice of mode of transportation of a commuter may be affected by the choices of one or more of the preceding commuters in the sequence. To achieve independence between rounds of play, we randomized the order of the players in the sequence in every round in our experiment. This procedure also prevented a given player from gaining information about the previous choices of any particular member of her group, and provided each player equal opportunity to assume all the possible positions in the sequence.

Choice Observability. Once the choice of transportation mode is conducted under the sequential protocol of play, the information that each player has when it is her turn to make a choice is critical. By contrast, simultaneous protocol setups impart zero information on choice 
observability among players within their experimental game. The significance of information in sequential decision-making settings is highlighted by the investigation of the effects of memory size in a sequential search problem with relative ranks known as the secretary problem (see, e.g., Ferguson, 1989, and Samuels. 1991 for literature reviews). In the secretary problem a decision maker (DM) observes a set of $n$ rankable applicants ( 1 being the best and $n$ the worst), who appear one at a time in random order with all the $n$ ! permutations equally likely. The absolute ranks of the $n$ applicants are not disclosed. Once the DM observes the $i^{\text {th }}$ applicant, she must decide either to accept (select) or reject it based on the observed rank of the applicant relative to all the applicants who preceded her in the sequence $(1 \leq i<n)$. In the rank minimization variant of the secretary problem (Chow et al., 1964), which is different from the more commonly known probability maximization variant, the DM objective is to minimize the expected (absolute) rank of the applicant selected. Chow et al. proved the astonishing result that as $n \rightarrow \infty$, the minimal expected rank of the selected applicant converges to 3.86. Rubin and Ferguson (1977) considered a generalization of the expected rank minimization problem under a severe information constraint, called the memory-length-one rule, where the DM may only consider (remember) just one of the previously observed applicants, and thereby be in a position to determine only whether the current applicant is better or worse than the currently remembered one. They proved an equally remarkable result that the limiting minimal expected rank may be kept finite even with such a severe constraint on information, and that its upper bound tends to 7.41 as $n \rightarrow \infty$.

Analogously to their results, all the choice options (transportation modes) in our traffic setup are available to each player, and choices are made sequentially under two conditions of choice observability. In the full choice observability condition, player $j$ has complete information about the transportation mode choices of all the players who preceded her in the sequence. Because the number of players in the group, $n$, is commonly known, the choice of the mode of transportation by player $j$ under full choice observability impacts the choices of players $j+1, j+2, \ldots, n$ who follow her in the sequence. Under the partial choice observability condition, which is examined by Rubin and Ferguson (1977) in the context of the secretary problem, each player is only informed of the choice made by the (single) player who preceded her in the sequence. Therefore, player $j$ does not know how many players who preceded her in the sequence chose ridesharing, nor may her decision directly affect the choices of players $j+2, j+3, \ldots, n$. Under both choice observability conditions, the first player in the sequence has no information at all, and the second player is only informed 
of the transportation mode choice of the first player. But starting with the third player in the sequence, the information sets of the remaining players under the two observability conditions diverge.

\section{The Directed Network Game in the Experiment}

\subsection{Description of the Network Game}

Figure 1 exhibits the choice task in our experiment. The task is visualized as a network with three edges representing three transportation modes, which share a common origin $O$ and common destination $D$. Formally, the network is modeled as a directed graph $G=(V, E)$ with a set $V$ of vertices (nodes), a set $E$ of edges (links), and a set $K \subseteq V \times E$ of origin-destination $(O, D)$ pairs. We consider a finite number $n$ of network users (players). The edge traffic $f_{i j}$ denotes the number of users choosing edge $(i \rightarrow j)$ that connects vertex $i$ with vertex $j$. The cost for each user traversing the edge $(i \rightarrow j)$, which is a function of $f_{i j}$, is denoted by $c_{i j}\left(f_{i j}\right)$. As it is commonly interpreted in transportation science, this cost term is an overall proxy for the time spent in traversing the edge, gasoline and oil consumed, and the externalities imposed by each network user on her group members. We assume that the cost of travel on any edge $(i \rightarrow j)$, given the amount of traffic on it, is the same for all $n$ users. The cost of travel for any given user is the sum of the edge costs traversed when the user travels from $O$ to $D$. Similar to previous research on strategic choice in traffic networks (e.g., Liu et al., 2015; Morgan et al., 2009; Rapoport et al., 2009), each user is assumed to choose a transportation mode that minimizes her cost of travel.

— Insert Fig. 1 around here -

Cost-sharing networks are frequently characterized in the previous literature by the nonlinear cost function $c_{i j}=a_{i j} / f_{i j}+b_{i j}$ for edge $(i \rightarrow j)$, with $a_{i j}, b_{i j} \geq 0$. In our experimental choice task displayed in Fig. 1, each of the two ridesharing transportation modes is also characterized by this type of non-linear cost function. For the Shuttle $(\mathrm{SH})$ transportation mode, $a_{i j}=150$ and $b_{i j}=0$; for the Carpool (CP) transportation mode, $a_{i j}=70$ and $b_{i j}=10$. If $a_{i j}$ is interpreted as the operating cost of the respective ridesharing transportation mode, then $\mathrm{SH}$ has a higher operating cost than $\mathrm{CP}$. However, $\mathrm{CP}$ has an additional fixed-cost term $\left(b_{i j}=10>0\right)$ that might be interpreted as, for example, the cost for the individual to park their private car at the carpool lot in order to travel 
with the carpool. The third transportation mode in Fig. 1, which we label as OD, has a linear cost function $c_{i j}=a_{i j} \times f_{i j}+b_{i j}$ where $a_{i j}=0$ and $b_{i j}=20$.

The two ridesharing transportation modes play different roles in our experimental setup. Firstly, they differ from each other in their degree of "riskiness", in the sense that the cost for a single player in choosing transportation mode $\mathrm{SH}$ is higher than the cost in choosing transportation mode CP, if no other (or a sufficiently small number of) players choose them. On the other hand, as we shall show, $\mathrm{SH}$ is an equilibrium strategy, which is also Pareto efficient, whereas CP is not. In contrast to the two ridesharing modes, the transportation mode OD is a riskless choice.

\subsection{Equilibrium Predictions}

We first construct subgame perfect equilibrium predictions for the full choice observability condition in the experimental network. We do so by means of backward induction.

For the $10^{\text {th }}$ player (the last one in the sequence), it is straightforward to show that if at least seven of the preceding players chose transportation mode $\mathrm{SH}$, then the player should also choose $\mathrm{SH}$ regardless of the choices of the preceding players. Inducing backward, numerical calculations comparing possible costs for the $9^{\text {th }}$ player show that, if at least six of the preceding players chose $\mathrm{SH}$, then the player should also choose $\mathrm{SH}$ (when the $10^{\text {th }}$ player would be expected to choose $\mathrm{SH}$ as well) in order to minimize cost of travel. Iterating this reasoning backward, the $3^{\text {rd }}$ player should choose transportation mode SH regardless of the choices of the preceding players; this means that the $3^{\text {rd }}$ to the $10^{\text {th }}$ players should all choose transportation mode $\mathrm{SH}$ along an equilibrium path. Consequently, the first two players should choose transportation mode $\mathrm{SH}$ as well under this equilibrium. As a result, under full choice observability, a subgame perfect equilibrium is for all players to choose the transportation mode $\mathrm{SH}$, which is also the welfare maximizing equilibrium. We call this the SH equilibrium.

A similarly styled argument might have been made to construct an alternative equilibrium path for all the players to choose transportation mode CP. However, because the cost of travel under this equilibrium is higher than that under the SH equilibrium, players early in the sequence who can trigger either equilibrium path, would strictly prefer choosing transportation mode SH to trigger the ensuing equilibrium. This is, indeed, one distinguishing potential advantage of full choice observability in coordinating players to achieve the welfare maximizing outcome; had the 
players been making choices with no information about each other's choices, there would have been three pure-strategy equilibria with all group members choosing either transportation mode $\mathrm{SH}, \mathrm{CP}$, or OD.

In deriving equilibrium predictions for the partial choice observability condition, we focus on pure-strategy equilibria in which a player's transportation mode choice is deterministic, given the transportation mode choice of the preceding player (if any). It is obvious that all the players choosing transportation mode $\mathrm{SH}$ is an equilibrium under the imitative strategy that a player chooses whatever the preceding player chose. In fact, this is also the unique equilibrium as long as the $5^{\text {th }}$ to $10^{\text {th }}$ players employ the imitative strategy, which, following the discussion earlier in this subsection, would then be able to replicate the equilibrium path of the SH equilibrium under full choice observability. We have not identified any other feasible pure-strategy equilibria under partial choice observability.

To conclude, the equilibrium predictions under the sequential protocol, in both the full and partial choice observability conditions, is for all the players to choose the ridesharing transportation mode SH. In contrast, when there is no choice observability (i.e., under the simultaneous protocol), there is a multiplicity of equilibria as mentioned above. The difference is driven by the possibility of multiple steps of backward induction under the sequential protocol. This process of game-theoretic backward induction reasoning might seem to be too artificial. In fact, it has been criticized both theoretically and experimentally, e.g., in the context of the centipede game. Theoretically, a serious criticism of backward induction involves a conceptual conundrum when the same player is called to act repeatedly within a game. This criticism does not apply to our present setup because each step in the backward induction is taken by a different player. As recently shown by Arieli and Aumann (2015), in a simple perfect information game, in which each player moves just once, backward induction outcomes must occur under common strong belief of rationality - a result that removes the theoretical conundrum.

But the theoretical validity of backward induction still hinges on assumptions about rationality. It thus remains important to examine the descriptive validity of predictions based on backward induction, such as our new predictions. If behavior approaches the predictions, it validates the game-theoretic backward induction reasoning in both the observability conditions. In addition, it would put our setup in stark contrast with Liu et al. (2015), who found multiplicity in 
equilibrium convergence both with their theoretical predictions and experimental data in a setup with zero choice observability (i.e., simultaneous protocol) among players. In particular, our partial choice observability condition only introduces a small degree of observability (the choice of the preceding player, if any). But apparently, if players condition their transportation mode choices on their observation, the result can be an effectively coordinated, welfare maximizing outcome. However, we might expect a longer path to coordination under partial (compared with full) observability, as we shall examine in our experimental data.

\section{Method}

\subsection{Subjects}

One hundred and eighty undergraduate students at the University of Arizona volunteered to participate in a group decision making experiment for payoff contingent on their performance. Male and female students participated in almost equal numbers. The participants (subjects) were divided into 18 groups of 10 members each. Nine groups participated in the network game under full choice observability (denoted as Condition FULL in subsequent discussion), and nine other groups under partial choice observability (denoted as Condition PART). On average, a session lasted about 75 minutes. Excluding a $\$ 5$ show-up bonus, the mean payment per subject across the two conditions was $\$ 22.00$.

\subsection{Procedure}

All the 18 sessions were conducted in a large computerized laboratory with multiple terminals situated in separate cubicles. Upon entering the laboratory, the subjects (players) were assigned randomly to their cubicles. They were handed written instructions that they read at their own pace (see the Appendix for the subject instructions in Condition PART). Questions about the experimental procedure were answered privately by the experimenter.

The instructions displayed the network in Fig. 1, explained the cost structure, and illustrated the computation of the cost over the edge(s) in each of the three transportation modes. Importantly, payoffs (rather than costs) were computed from the equation: Payoff $=\$ 90 / \mathrm{cost}$. The payoffs were computed over five rounds (out of a total of 50 rounds) which were selected randomly at the end of the session.

Under both conditions, the subject was asked to choose and subsequently submit one of the three transportation modes (see table in the lower right-hand corner of Fig. 1). Before 
confirming her choice, she was given the option of changing it as often as she wished. Transportation mode choices were made by clicking a button in a list of the three transportation modes. In both conditions, subjects were assigned randomly to one of the 10 positions in the sequence (see Appendix), which appeared on the screen as 10 red dots in a line. A transportation mode choice caused the color of the dot to change from red to green. When it was her turn to move, each subject in Condition FULL was informed of the transportation mode choices of all the subjects who preceded her in the sequence (if any), where a subject in Condition PART was only informed of the choice made by the subject who immediately preceded her (if any). Once all the 10 group members submitted their transportation mode choices, a new screen was displayed informing each subject about (1) the present round number, $j$; (2) the transportation mode that this subject had chosen on round $j-1$ (if $j>1$ ); and (3) the subject's payoff for round $j-1$ (if $j>1$ ). Note that although order of play was randomized among players in each round, the "randomization" was constrained so that during the session each player was assigned the first position in the sequence in exactly five rounds of play.

\section{Results}

This section presents a comparison of the two choice observability conditions, analysis of the behavioral dynamics of play over iterations of the stage game, analysis of the differences between groups in each of the two conditions, and a similar analysis of the differences between group members.

\subsection{Treatment Effects}

To examine the effects of the degree of choice observability, Figs. 2A and 2B exhibit all the raw data by condition, group, round of play, and individual choices by the order that they were submitted. Figures 2A and 2B display the raw data for Conditions FULL and PART, respectively. Each figure includes nine panels, one for each group. The 50 rows indicate all the rounds of play, and the 10 columns the exogenously determined order in the sequence (and not the subject number). Each cell in the 50 by 10 display is shaded either dark grey for the individual choice of transportation mode $\mathrm{SH}$, light grey for the individual choice of transportation mode $\mathrm{CP}$, or white (blank) for the individual choice of transportation mode OD. For example, in round 1 of Group F1 (Fig. 2A), each of the first five players in the sequence chose transportation mode $\mathrm{SH}$, the sixth 
player chose transportation mode OD, and the remaining four players chose transportation mode SH. Figure 2A shows that Groups F4, F7, and F9 in Condition FULL converged to the equilibrium in at most 10 rounds of play. With two exceptions of Player 1 in Group F9, the players in these three groups did not deviate from equilibrium during the last 40 rounds of play. Groups F2, F5, F8, and F6 seem to have converged to equilibrium in the final 10 rounds of play. Two additional groups in this condition do not seem to have converged to any of the three transportation modes.

— Insert Figs. 2A, 2B, and 3 around here -

Figure 2B shows that only a single group (Group P4) converged to equilibrium in fewer than 10 rounds in Condition PART. Groups P1, P6, P8, and P9 also converged to equilibrium, although it took them longer to do so. Group P7 seems to have converged to the choice of the private transportation mode OD after 32 rounds, and Groups P2, P3, and P5 do not seem to have converged to any of the three transportation modes. Correspondingly, as shown in Fig. 3, mean group costs in both conditions declined across the 50 rounds with a general trend approaching equilibrium.

To formalize these observations, we define convergence to equilibrium as follows: a group is said to converge to equilibrium if in 14 of the final 15 rounds of play all the 10 group members chose transportation mode SH. According to this definition, six groups in Condition FULL and five in Condition PART achieved convergence. This provides partial support to the main hypothesis of no difference between the two choice observability conditions. However, a further inspection of the raw data in Figs. 2A and 2B suggests that the out-of-equilibrium choices differed from one condition to another with more group members choosing transportation mode $\mathrm{SH}$ in Condition FULL than in Condition PART. To verify this observation, we counted the number of choices by transportation mode and condition, which are presented in Table 1. A chi-square test rejects the null hypothesis that the choice of transportation mode is independent of the condition of choice observability $\left(\chi^{2}(2)=997, N=9000, p<0.001\right)$. Table 1 shows that the players in Condition FULL were more likely to choose transportation mode SH (3450 observations) than players in Condition PART (2137 observations). Conversely, the players in Condition PART were three times more likely to choose the private transportation mode OD than the players in Condition FULL. 
To further examine the effects of the degree of choice observability, experience gained in playing the iterated ridesharing game, and their interaction, we recorded the number of choices of transportation mode SH as the dependent measure, and conducted a condition by block $(2 \times 10)$ mixed-design ANOVA test with block (a set of five contiguous rounds) as the repeated measure, and group as the unit of analysis. The ANOVA yielded two significant main effects due to condition $(F(1,16)=27.3, p<0.001)$ and block $(F(9,144)=2.04, p<0.05)$; the condition by block interaction effect was not significant $(p>0.25)$. Consistent with Table 1 and the related chi-squared test result, the ANOVA shows that players in Condition FULL were more likely to choose the transportation mode SH than players in Condition PART, and that in both conditions the frequency of choices of SH increased with experience (see the right-most column in Table 2).

\section{— Insert Table 2 around here -}

\subsection{Dynamics of Play}

Inspection of the raw data in Figs. 2A and 2B shows that the individual groups in each condition differed markedly from one another in: (1) whether they converged to equilibrium, (2) how quickly they converged to equilibrium, if at all, and (3) the sequential dependencies within and between rounds of play. To better understand the effects of these differences, we proceeded with a more detailed analysis of the transition frequencies. For each group separately, we counted the number of $\mathrm{SH}, \mathrm{CP}$, and $\mathrm{OD}$ choices that immediately followed the choice of one of these three transportation modes by the preceding (and different) player in the sequence. Note that because of randomization of the sequences on each round of play, the identities of these players differed from one round to another. Omitting the final player in the sequence, we thus constructed for each group a $3 \times 3$ transition matrix of conditional frequencies (hereby called transitions) with $450(50 \times 9)$ entries. Tables $3 \mathrm{~A}$ and $3 \mathrm{~B}$ present the results for Conditions FULL and PART, respectively. For example, consider the transition matrix for Group F1 in Table 3A. For this group, we recorded a total of 95, 153, and 202 choices of transportation modes SH, CP, and OD, respectively. A choice of transportation mode SH by player $k$ in the sequence $(k=1,2, \ldots, 9)$ was immediately followed by a choice of either transportation mode $\mathrm{SH}, \mathrm{CP}$, or OD on 44, 17, and 34 occasions, respectively. Similarly, a choice of transportation mode CP was immediately followed by a choice of either transportation mode $\mathrm{SH}, \mathrm{CP}$, or $\mathrm{OD}$ on 20,111 , and 22 times, respectively. A choice of transportation mode OD was immediately followed by a choice of either transportation mode SH, 
$\mathrm{CP}$, or $\mathrm{OD}$ on 25, 24, and 153 times. Below each panel, we report the number of transitions in the three cells above the main diagonal, the three cells on the main diagonal, and three more cells below the main diagonal. The first number indicates a switch to a less risky transportation mode (in the sense discussed in Section 2.1), the second indicates no switch at all, while the third indicates a switch to a riskier transportation mode.

- Insert Tables 3A and 3B around here -

Using three separate $t$-tests, we then compared to each other the two conditions in terms of the transition frequencies above, on, and below the main diagonal. None of these tests yielded significant differences, suggesting that the mean number of decisions that did not change from the previous transportation mode choice, switched towards a less risky transportation mode choice, or switched towards a riskier transportation mode choice, did not vary by condition.

\subsection{Individual-Level Analysis}

For a different perspective on the behavioral dynamics of play, we re-examined some of the measures reported in the preceding section. We did so by computing for each player the number of transportation mode choices that were

(1) identical to the transportation mode choice made by the player who preceded her in the sequence (if any);

(2) less risky than the transportation mode choice made by the player who preceded her, where SH $>\mathrm{CP}>\mathrm{OD}$ in terms of riskiness following earlier discussion; and

(3) more risky than the transportation mode choice made by the player who preceded her.

We also examined

(4) the mean travel cost per player per round; and

(5) the number of $\mathrm{SH}$ choices that each player made when assigned the first position in the sequence, i.e., $k=1$.

Recall that the randomization of the order of play was constrained so that each player was assigned the first position in the sequence in exactly five rounds of play; hence, this measure had an upper limit of 45 for each player. 
Several of these measures were reported earlier at the condition and group level. Here, we computed them at the individual level in order to obtain results that might help in explaining individual deviations from equilibrium play.

— Insert Table 4 around here -

The first panel of Table 4 presents the mean number of transportation mode choices that were identical to the preceding choice, if any, in the sequence. Standard deviations (s.d.) are shown in parentheses. The range indicates the extent of individual differences for this measure. For example, players in Condition FULL averaged 40.80 decisions (out of 45) that were the same as the preceding choice. Examination of the range shows that a single player made only 10 decisions that were identical to the preceding choice, whereas several players repeated their preceding choice on all the 45 rounds when in position $k>1$. Consistent with the data presented above at the group level (Tables 3A and 3B), the mean difference between the two observability conditions was not significant.

The next two panels of Table 4 report measures of the direction of the switch in transportation mode choice toward either a more risky or less risky alternative compared with the preceding choice in the sequence. Substantial differences are noted for these two measures. On average, 2.19 and 3.08 transportation mode choices switched in the direction of a less risky alternative in Conditions FULL and PART, respectively. Further individual-level analysis (not reported in Table 4) reveals that 25 and 27 players in Conditions FULL and PART, respectively, never switched to a less risky alternative. The highest number of individual switches is 16 and 19 (again, out of 45), in Conditions FULL and PART, respectively. Examination of the switches in the direction of riskier transportation mode choice (panel 3) shows means of 2.01 and 2.61 in Conditions FULL and PART, respectively. Individual results show that a single player made 27 decisions in the direction of a riskier choice in Condition FULL. For Condition PART, we find a single subject who made 30 such decisions. Further examination shows that 30 and 32 players (out of 90) never switched to a riskier alternative in Conditions FULL and PART, respectively. Consistent with the data presented above at the group level, the mean difference between the less risky or riskier transitions is not significant.

The mean individual travel cost per round (panel 4) was 18.54 and 22.22 for Conditions FULL and PART, respectively. This difference between the two conditions is significant $(t(178)$ 
$=3.05, p<0.01$ ), and also appears in Fig. 3 as having persisted towards the end of the session. Finally, in panel 5, the mean number of SH transportation mode choices in position $k=1$ was 3.90 and 2.70 (out of 5) in Conditions FULL and PART, respectively. This difference between the two means is significant $(t(178)=4.66, p<0.001)$, indicating that players with full choice observability were more likely to initiate a round by choosing SH than players with partial choice observability. Further examination of individual results reveals that 41 and 26 subjects always began a round by choosing SH in the Conditions FULL and PART, respectively, thereby nudging the other group members to choose ridesharing. This compares to 5 and 22 subjects in Conditions FULL and PART, respectively, who never chose transportation mode SH when it was their turn to start a round, possibly because they considered this decision to be too risky or had doubts about the rationality of some of the group players.

\section{Discussion}

In this study, we investigate the coordination problems faced by traffic network users in the presence of ridesharing opportunities. The coordination problems arise because of the positive externalities in the sharing of cost through ridesharing. We focus on how sequential observability of transportation mode choices may prove to be a powerful facilitator of coordination in this context.

We capture the essence of the issues by a simple directed network game with experimentally testable predictions. Our experimental setup calls for tacit coordination in small groups under two alternative information structures. Members of each group in each of the two information structures are asked to choose one of three alternative modes of transportation from a common origin to a common destination. The costs of travel differ from one transportation mode to another, and they are commonly known. One of the transportation modes (called the private transportation mode) has a fixed cost of travel; a group member who chooses this transportation mode assumes no risk and imposes no externality on other group members choosing the same transportation mode. Two other transportation modes (called ridesharing transportation modes) each imposes a positive externality as the cost of traversing this transportation mode is shared equally by all the group members, if any, who choose it. The two ridesharing transportation modes play different roles in our experimental setup. They differ from each other in their degree of "riskiness" in the sense that the cost for a single player in choosing one of them (transportation 
mode $\mathrm{SH}$ ) is higher than the cost of the other (transportation mode $\mathrm{CP}$ ), if no other (or a sufficiently small number of) players choose these transportation modes. On the other hand, transportation mode $\mathrm{SH}$ is an equilibrium strategy, which is also Pareto efficient, whereas transportation mode $\mathrm{CP}$ is not. Because the choice of transportation mode $\mathrm{CP}$ is less costly, it may serve as an intermediate stage in nudging the players to share their ride without risking too much. Figures $2 \mathrm{~A}$ and $2 \mathrm{~B}$ jointly show that while no group in our experiment converged to the choice of transportation mode $\mathrm{CP}$, this transportation mode was instrumental in inducing many players to switch from the choice of the riskless private transportation mode OD to the choice of the riskier but potentially more profitable transportation mode $\mathrm{SH}$.

In contrast to most previous studies on strategic choices in traffic networks (see Rapoport and Mak, in press), choices in our experiment were made sequentially, not simultaneously. Our study compares two alternative sequential protocols of play. Under one protocol (Condition FULL), each group member is fully and accurately informed of the choices of all the group members who preceded her in the (exogenously determined) sequence. Under the other protocol (Condition PART), her information is limited to the choice of the single group member who preceded her immediately in the sequence. The former protocol allows for a relatively high degree of tacit communication of choices among players; when choosing her transportation mode, a player knows that her decision will be revealed to the group members who are yet to make their decisions, and therefore her choice may influence their future behavior. Under the latter protocol, with limited choice observability, communication of choice is limited to the next player in the sequence, and the history of play is restricted to the choice of the previous player.

Convergence to the equilibrium solution, which is theoretically invariant under the two information structures implemented in our experiment, depends on optimal best responses of all the group members under backward induction. As is evident from a close inspection of the raw data in Figs. 2A and 2B, most of our subjects found the ridesharing game quite daunting. It is evident that convergence is only likely with multiple iterations of the stage game. Moreover, in our experiment, randomization of the order of play in each round must have slowed down the convergence process considerably. Yet, under a strict definition of convergence, 11 of the 18 groups did converge to equilibrium, whereas the remaining seven groups did not. Thus, a major finding of our experiment is that, regardless of the level of choice observability, given enough 
iterations of the stage game our subjects found ways to overcome the challenge of coordinating their choices, and thereby maximize their payoffs. Our results also show that, although the information structure had no significant effect on the number of groups that achieved convergence, it had a significant effect on the behavioral dynamics of play. With limited observability, achieving coordination of all the group members took longer and was consequently costlier (Table 4).

To sum up, our experimental evidence, in line with theoretical analysis, shows that even a limited extent of sequential choice observability might lead to efficient coordination in ridesharing. This should especially be viewed in contrast with the study of Liu et al. (2015), who examined the zero-choice observability (i.e., simultaneous protocol) version of our setup, and found that the multiplicity of equilibria in theory was manifested in a similar divergence in behavioral patterns.

Our study abstracts the essential issues of coordination in ridesharing into a directed network game with experimentally testable predictions. The advantage of laboratory experimentation is rigorous control, so that we can be confident of the internal validity of our results. Meanwhile, despite the level of abstraction of the experimental task, we consider that similar decision scenarios in real life would be characterized by the same essential features, including information provision in the form of varying levels of observability. Moreover, partial observability in our experiment could be seen as a highly impoverished, in fact almost minimal, form of information provision. As such, our findings - that efficiency could be achieved even in such a case - suggest applicability to richer forms of information provision in real-life contexts.

A caveat of our findings is that, in terms of behavioral dynamics, convergence to efficiency would be slower with more limited observability, resulting in a significant increase in travel cost. In the age of social media, large-scale communication of choices could be brought about instantly, and our results suggest that ridesharing has a high potential in achieving socially desirable outcomes. Nevertheless, it would be useful to further encourage network users to share network choice information, which could then accelerate the behavioral dynamics to achieve even higher welfare gains over time.

Subsequent research could be developed along this direction, such as investigating endogenous transportation mode choice communications among users. An interesting aspect of endogenous communication is that it can be "cheap talk" without an incentive-compatible rationale for the communicator to tell the truth. As such, in terms of actual behavior, if endogenous 
communication can be used as a coordination device, it may truthfully be employed with a significant influence on choices (see Mak et al., 2015, for a related investigation in a different context). Alternatively, given that traffic networks could be subject to exogenous uncertainties, future extensions could look at how sequential choice observability might interact with exogenous uncertainties in its ability to maintain a high level of coordination among network users. 


\section{References}

Acemoglu, D., Makhdoumi, A., Malekian, A., and Ozdagar, A. (2016). Informational Braess' Paradox: The effect of information on traffic congestion. arXiv: 1601.02039v1.

Agatz, N. A. H., Erera, A. L., Savelsbergh, M. W. P., and Wang, X. (2012). Optimization for dynamic ride-sharing: A review. European Journal of Operational Research, 223, 295-303.

Anshelevitch, E., Dasgupta, A., Kleinberg, J., Tardos, É., Wexler, T., and Roughgarden, T. (2008). The price of stability for network design with fair cost allocation. SIAM Journal of Computing, $\underline{\text { 38, }}$, 1602-1623.

Arieli, I. and Aumann, R. J. (2015). The logic of backward induction. Journal of Economic Theory, 159, 443-464.

Berbeglia, G., Cordeau J.-F., and Laporte, G. (2010). Dynamic pickup and delivery problems. European Journal of Operational Research, 202, 8-15.

Charness, G., Ferl, F., Meléndez-Jiménez, M. A., and Sutter, M. (2014). Experimental games on networks: Underpinnings of behavior and equilibrium selection. Econometrica, $\underline{\mathbf{8 2}}$, 1615-1670.

Chen, H.-L., Roughgarden, T., and Valiant, G. (2010). Designing network protocols for good equilibria. SIAM Journal of Computing, $\underline{\mathbf{3 9}}$, 1799-1832.

Chen, H.-L. and Chen, Y. (2011). The potential of social identity for equilibrium selection. American Economic Review, 101, 2562-2589.

Chow, Y. S., Moriguti, S., Robbins, H. and Samuels, S. M. (1964). Optimal selection based on relative rank (the "secretary problem"). Israel Journal of Mathematics, $\underline{\mathbf{2}}$, 81-90.

Crawford, V. P. (1995). Adaptive dynamics in coordination games. Econometrica, $\underline{\mathbf{6 3}}, 103-$ 144.

Erev, I. and Rapoport, A. (1998). Coordination, "magic", and reinforcement learning in market entry games. Games and Economic Behavior, $\underline{\mathbf{2 3}}, 313-325$.

Ferguson, T. S. (1989). Who solved the secretary problem? Statistical Science, $\underline{\mathbf{4}}, \mathbf{2 8 2 - 2 8 9 .}$ 
Furuhata, M., Dessouky, M., Ordonez, F., Brunet, M., Wang, X., and Koenig, S. (2013). Ridesharing: The state-of-the-art and future directions. Transportation Research Part B: Methodological, $\underline{\text { 57, }}$,28-46.

Gisches, E. and Rapoport, A. (2012). Degrading network capacity may improve performance: Information effects in the Braess paradox. Theory and Decision, $\underline{\mathbf{7 3}}, 267-293$.

Goeree, J. and Holt, C. (2015). An experimental study of costly coordination. Games and Economic Behavior, 51, 349-364.

Hoefer, M. (2013). Strategic cooperation in cost sharing games. International Journal of Game Theory, 느, 29-53.

Jain, K. and Mahdian, M. (2007). Cost sharing. In N. Nisan, T. Roughgarden, É Tardos, and V. Vazirani (Eds.), Algorithmic Game Theory, pp. 385-410. Cambridge: Cambridge University Press.

Liu, C., Mak, V., and Rapoport, A. (2015). Cost-sharing in directed networks: Experimental study of equilibrium choice and system dynamics. Journal of Operations Management, 39-40, 3147.

Mak, V., Zwick, R., Rao, A. R., and Pattaratanakun, J. A. (2015). "Pay what you want" as threshold public good provision. Organizational Behavior and Human Decision Processes, $\underline{\mathbf{1 2 7}}$, $30-43$.

Morgan, J., Orzen, H., and Sefton, M. (2009). Network architecture and traffic flow: Experiments on the Pigou-Knight-Downs and Braess paradoxes. Games and Economic Behavior, 66, 348-372.

Moulin, H. (2002). The proportional random allocation of indivisible units. Social Choice and $\underline{\text { Welfare, }} \underline{\mathbf{1 9}}, 381-413$.

Rapoport, A., Gisches, E., and Mak, V. (2014). Distributed decisions in networks: Laboratory study of routing splittable flow. Production and Operations Management, 23, 314-331. 
Rapoport, A., Kugler, T., Dugar, S., and Gishces, E. (2009). Choice of routes in congested traffic networks: Experimental tests of the Braess paradox. Games and Economic Behavior, $\underline{\mathbf{6 5}}$, $538-571$.

Rapoport, A. and Mak, V. (in press). Strategic interaction in transportation networks. In K. Donohue, E. Katok, and S. Leider (Eds.), Handbook of Behavioral Operations, New York: Wiley.

Rapoport, A., Mak, V., and Zwick, R. (2006). Navigating congested networks with variable demand: Experimental evidence. Journal of Economic Psychology, 27, 648-666.

Rubin, H. and Samuels, S. M. (1977). The finite-memory secretary problem. The Annals of Probability, 5, 627-635.

Samuels, S. M. (1991). Secretary problems. In P. K. Sen (Ed.), Handbook of Sequential Analysis, Boston: Marcel Dekker, pp. 381-405.

Schelling, T. C. (1960). The Strategy of Conflict. Cambridge, MA: Harvard University Press.

Seale, D. A. and Rapoport, A. (2000). Elicitation of strategy profiles in large group coordination games. Experimental Economics, $\underline{\mathbf{3}}, 153-179$.

Selten, R., Chmura, T., Pitz, T., Kube, S., and Schreckenberg, M. (2007). Commuters route choice behavior. Games and Economic Behavior, $\underline{\mathbf{5 8}}$, 394-406.

Shubik, M. (1982). Game Theory in the Social Sciences, Vol. 1. Cambridge MA: MIT Press.

Syrgkanis, V. (2010). The complexity of equilibria in cost sharing games. Internet and Network Economics, pp. 366-377.

Van Huyck, J. B., Battalio, R. C., and Beil, R. O. (1990). Tacit coordination games, strategic uncertainty, and coordination failure. American Economic Review, 우, 234-248.

Van Huyck, J. B., Battalio, R. C., and Beil, R. O. (1991). Strategic uncertainty, equilibrium selection, and coordination failure in average opinion games. Quarterly Journal of Economics, $\underline{\mathbf{9 1}}$, 885-910.

Young, H. P. (1994). Cost allocation. In R. Aumann and S. Hart (Eds.), Handbook of Game Theory with Economic Applications, Vol. 2, Ch. 34, pp. 1194-1235. 
Young, H. P. (1985). Producer incentives in cost allocation. Econometrica, 53, 757-765.

Young, H. P. (1988). Cost allocation, demand revelation, and core implementation. Mathematical Social Sciences, $\underline{\text { 36}}$, 213-228. 
Fig. 1. The Transportation Mode Choice Task in the Experimental Game

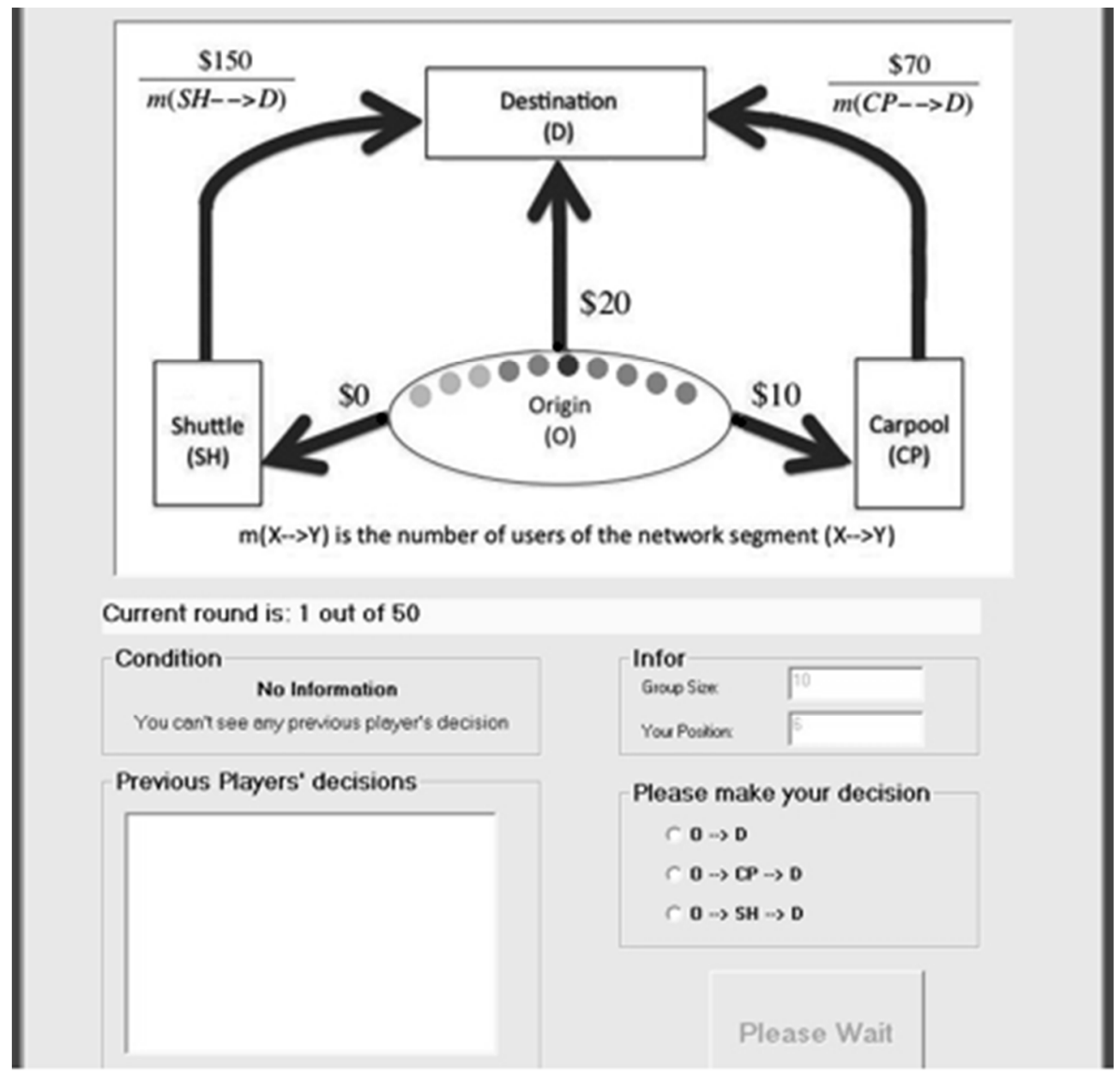


Fig. 2A. Transportation Mode Decisions by Group and Round - Condition FULL Order of Play $(k)$

Order of Play $(k)$

Order of Play $(k)$
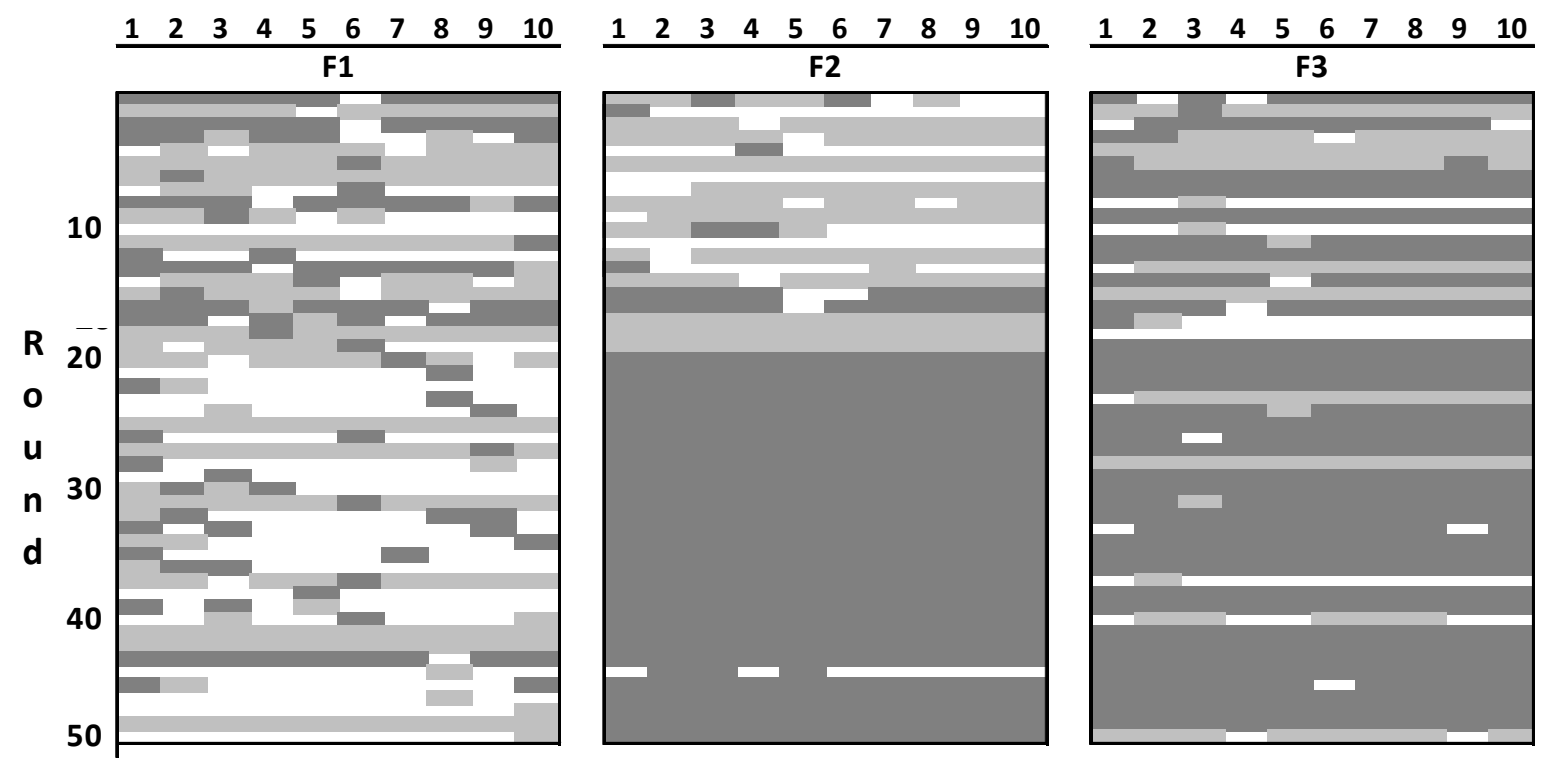

F4

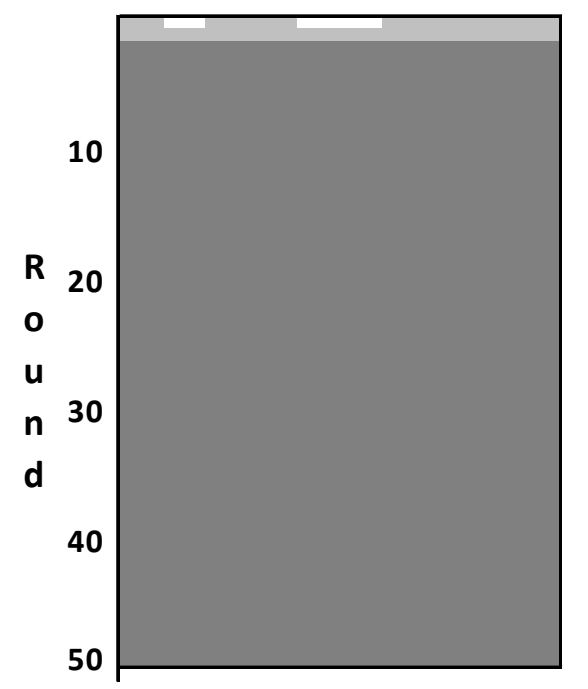

F5

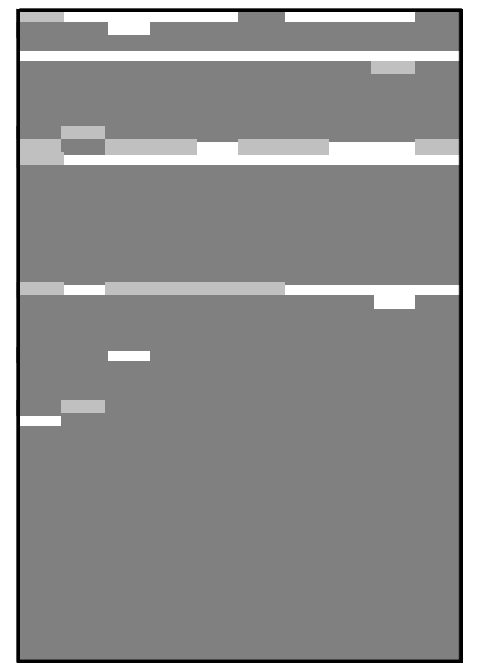

F6

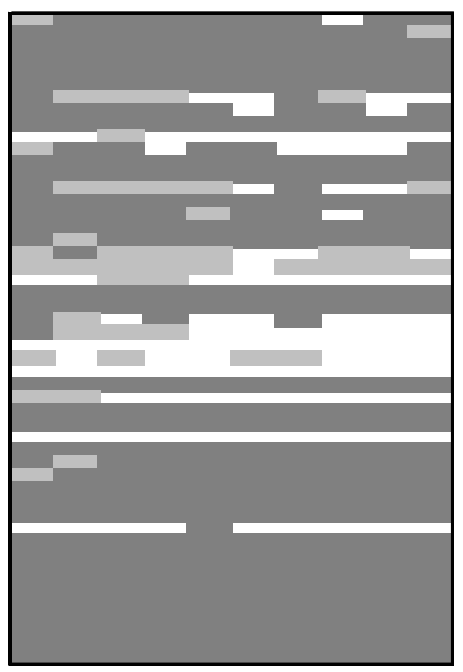

F8

F9
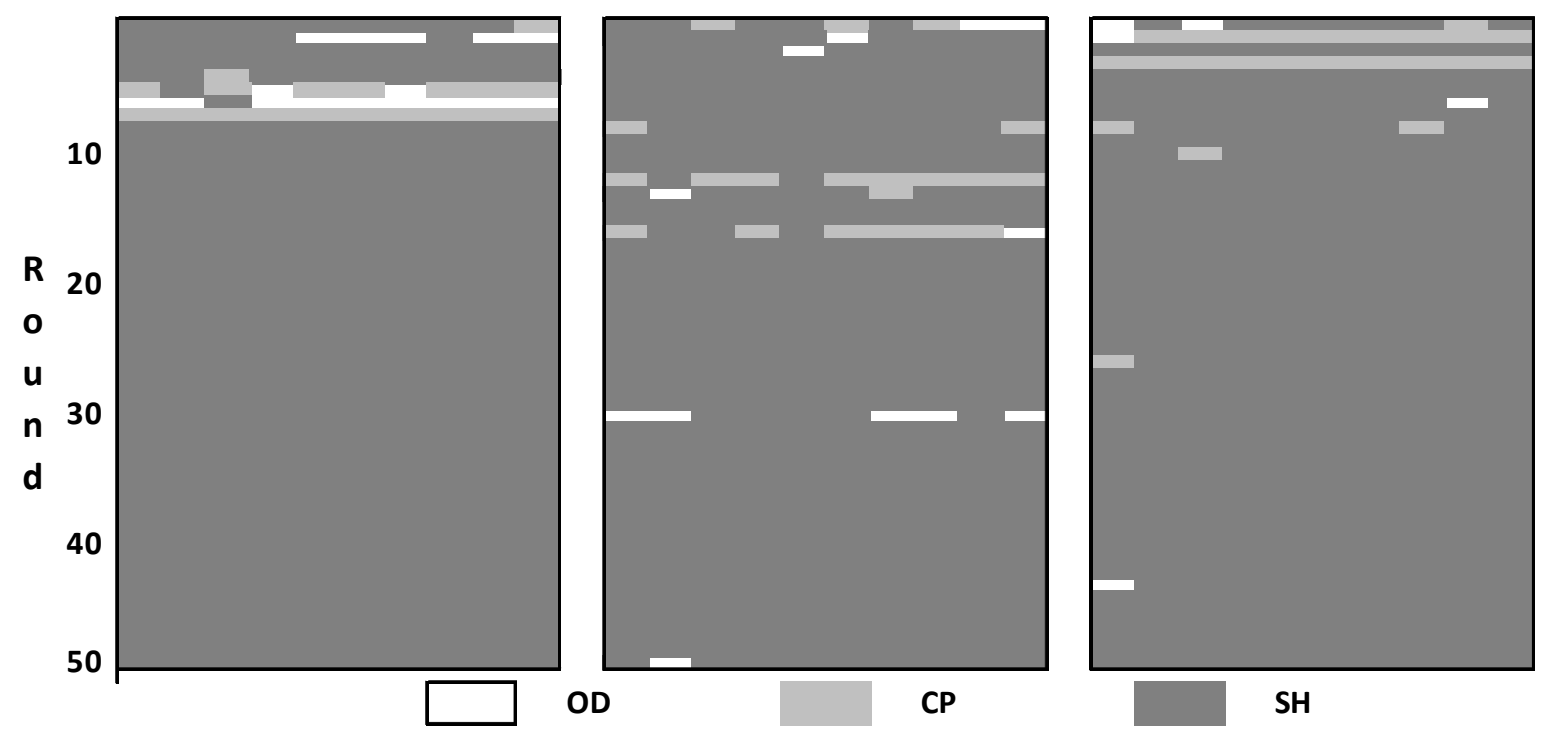
Fig. 2B. Transportation Mode Decisions by Group and Round - Condition PART

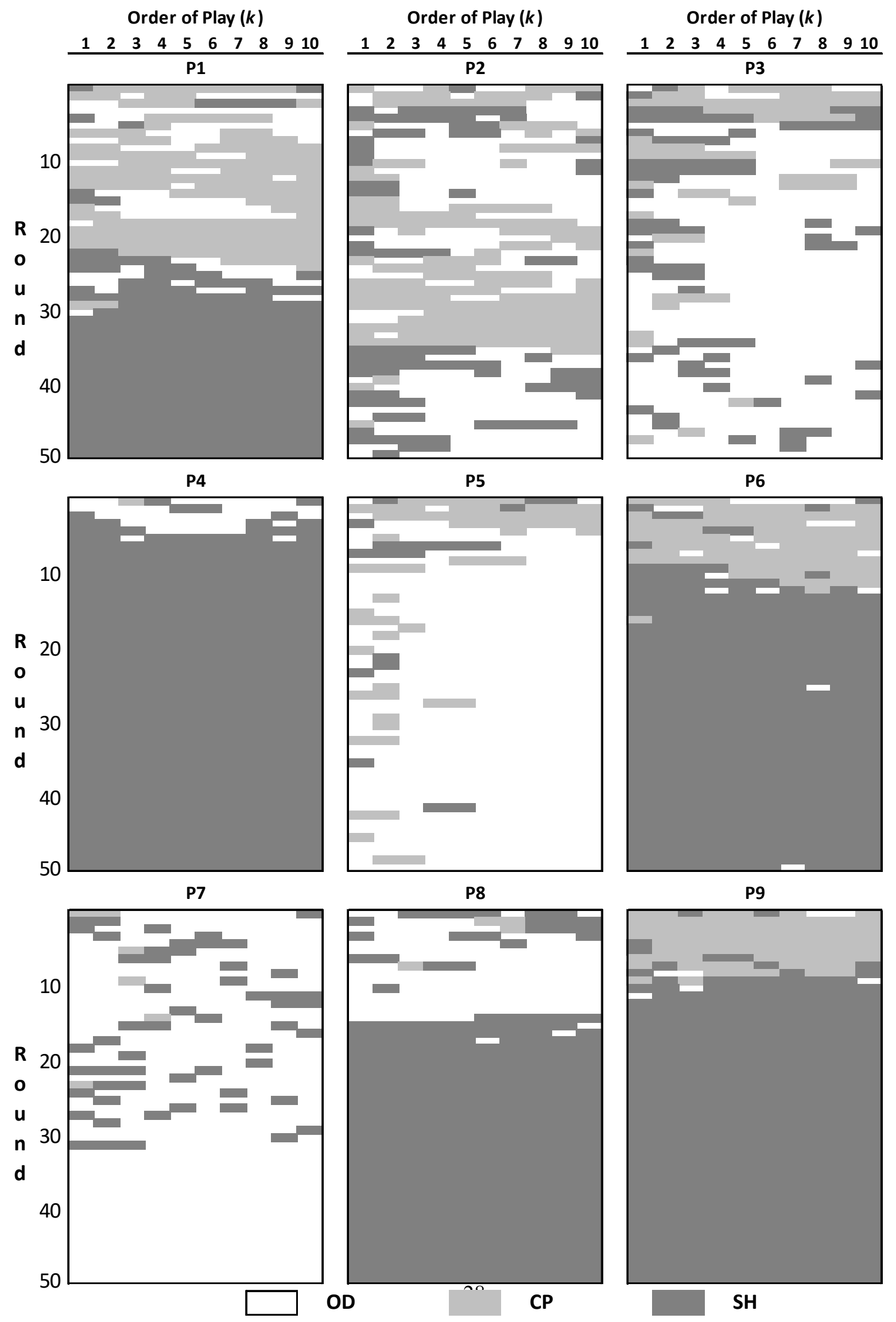


Fig. 3. Mean Group Cost (\$) by Round and Condition. The Gray Dashed Lines Indicate the Group Costs When All Users Choose SH (\$150), CP (\$170), and OD (\$200), Respectively.

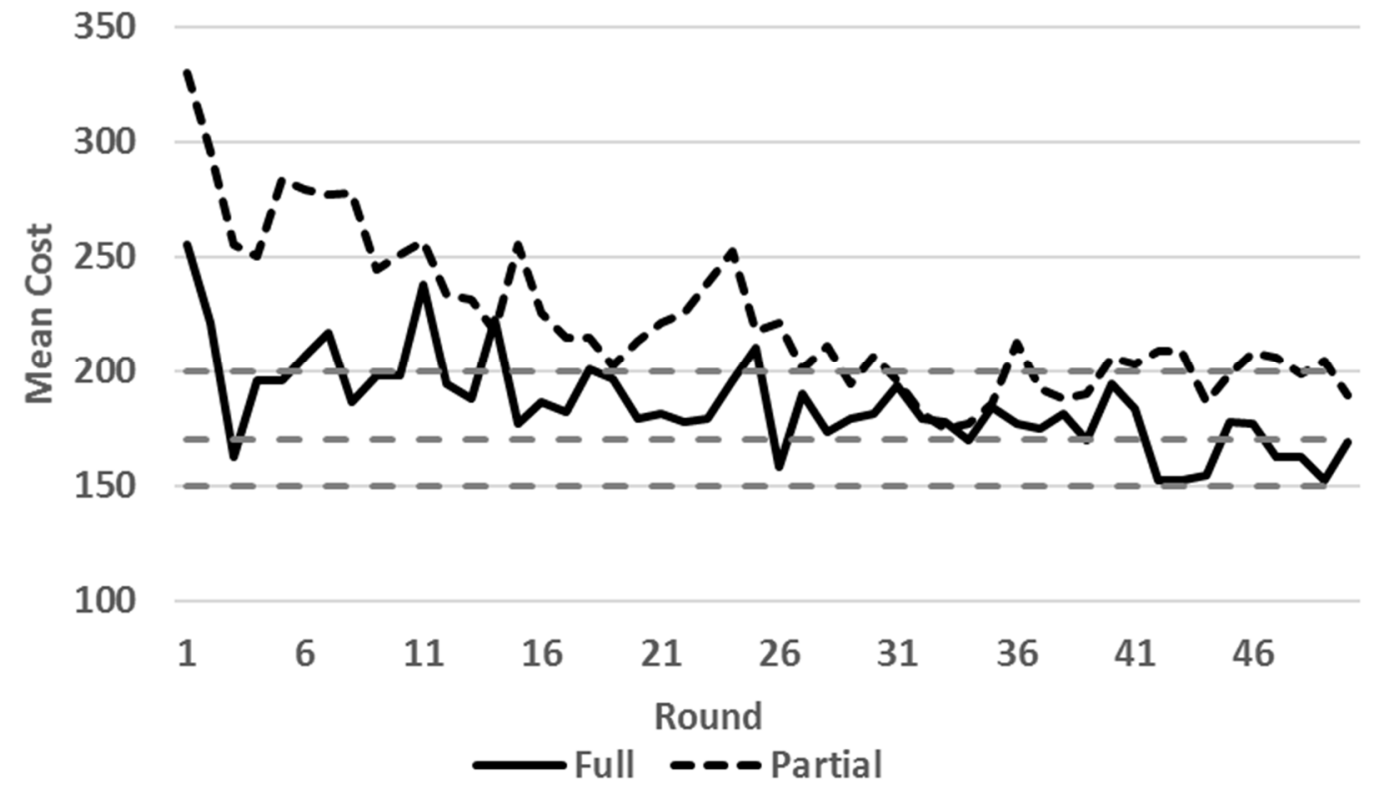


Table 1. Frequency of Choice of Transportation Mode by Condition

\begin{tabular}{|cccc|}
\hline & \multicolumn{3}{c|}{ Number of Decisions } \\
\cline { 2 - 4 } Condition & SH & CP & OD \\
\hline FULL & 3450 & 514 & 536 \\
PART & 2137 & 605 & 1758 \\
\hline
\end{tabular}

Table 2. Mean Number of SH Choices by Condition, Group, and Block

\begin{tabular}{|c|c|c|c|c|c|c|c|c|c|c|}
\hline \multicolumn{10}{|c|}{ Condition FULL } & \multirow[b]{2}{*}{ Mean } \\
\hline $\begin{array}{l}\text { Rounds in } \\
\text { Block }\end{array}$ & $\mathrm{F} 1$ & $\mathrm{~F} 2$ & F3 & $\mathrm{F} 4$ & F5 & F6 & F7 & $\mathrm{F} 8$ & F9 & \\
\hline $1-5$ & 4.6 & 0.8 & 3.8 & 6.0 & 6.0 & 9.4 & 8.6 & 8.6 & 5.4 & 5.9 \\
\hline $6-10$ & 2.4 & 0.0 & 6.4 & 10.0 & 9.8 & 6.0 & 4.4 & 9.6 & 9.4 & 6.4 \\
\hline $11-15$ & 2.4 & 0.6 & 5.6 & 10.0 & 6.2 & 7.4 & 10.0 & 8.0 & 9.8 & 6.7 \\
\hline $16-20$ & 3.6 & 3.4 & 4.0 & 10.0 & 10.0 & 5.6 & 10.0 & 8.6 & 10.0 & 7.2 \\
\hline $21-25$ & 1.0 & 10.0 & 7.8 & 10.0 & 7.8 & 4.8 & 10.0 & 10.0 & 10.0 & 7.9 \\
\hline $26-30$ & 1.0 & 10.0 & 7.8 & 10.0 & 9.8 & 2.0 & 10.0 & 10.0 & 9.8 & 7.8 \\
\hline $31-35$ & 2.0 & 10.0 & 9.4 & 10.0 & 9.6 & 7.8 & 10.0 & 9.0 & 10.0 & 8.6 \\
\hline $36-40$ & 1.6 & 10.0 & 8.0 & 10.0 & 10.0 & 8.0 & 10.0 & 10.0 & 10.0 & 8.6 \\
\hline $41-45$ & 2.0 & 8.6 & 8.0 & 10.0 & 10.0 & 10.0 & 10.0 & 10.0 & 9.8 & 8.7 \\
\hline $46-50$ & 0.4 & 10.0 & 7.8 & 10.0 & 10.0 & 10.0 & 10.0 & 9.8 & 10.0 & 8.7 \\
\hline Overall & 2.1 & 6.3 & 6.9 & 9.6 & 8.9 & 7.1 & 9.3 & 9.4 & 9.4 & 7.7 \\
\hline \multicolumn{11}{|c|}{ Condition PART } \\
\hline & \multicolumn{9}{|c|}{ Group } & \\
\hline $\begin{array}{l}\text { Rounds in } \\
\text { Block }\end{array}$ & $\mathrm{P} 1$ & $\mathrm{P} 2$ & P3 & $\mathrm{P} 4$ & P5 & P6 & $\mathrm{P} 7$ & P8 & P9 & Mean \\
\hline $1-5$ & 1.4 & 2.8 & 2.6 & 3.2 & 1.0 & 1.4 & 2.0 & 3.6 & 0.6 & 2.1 \\
\hline $6-10$ & 0.2 & 2.0 & 1.8 & 9.6 & 1.6 & 1.0 & 1.4 & 0.8 & 3.2 & 2.4 \\
\hline $11-15$ & 0.2 & 1.6 & 2.6 & 10.0 & 0.0 & 7.2 & 1.6 & 1.2 & 9.6 & 3.8 \\
\hline $16-20$ & 0.4 & 0.2 & 1.4 & 10.0 & 0.0 & 9.8 & 1.6 & 9.4 & 10.0 & 4.8 \\
\hline $21-25$ & 2.0 & 1.4 & 1.6 & 10.0 & 0.6 & 10.0 & 2.0 & 10.0 & 10.0 & 5.3 \\
\hline $26-30$ & 6.4 & 0.0 & 0.6 & 10.0 & 0.0 & 9.8 & 1.6 & 10.0 & 10.0 & 5.4 \\
\hline $31-35$ & 9.8 & 0.0 & 0.6 & 10.0 & 0.0 & 10.0 & 0.8 & 10.0 & 10.0 & 5.7 \\
\hline $36-40$ & 10.0 & 4.4 & 1.6 & 10.0 & 0.2 & 10.0 & 0.0 & 10.0 & 10.0 & 6.2 \\
\hline $41-45$ & 10.0 & 2.2 & 1.0 & 10.0 & 0.4 & 10.0 & 0.0 & 10.0 & 10.0 & 6.0 \\
\hline $46-50$ & 10.0 & 2.4 & 1.2 & 10.0 & 0.0 & 9.8 & 0.0 & 10.0 & 10.0 & 5.9 \\
\hline Overall & 5.0 & 1.7 & 1.5 & 9.3 & 0.4 & 7.9 & 1.1 & 7.5 & 8.3 & 4.7 \\
\hline
\end{tabular}


Table 3A. Transition Frequencies by Group: Condition FULL

\begin{tabular}{r|ccc|}
$\mathrm{F} 1$ & $\mathrm{SH}_{k+1}$ & $\mathrm{CP}_{k+1}$ & $\mathrm{OD}_{k+1}$ \\
\cline { 2 - 4 } $\mathrm{SH}_{k}$ & 44 & 17 & 34 \\
$\mathrm{CP}_{k}$ & 20 & 111 & 22 \\
$\mathrm{OD}_{k}$ & 25 & 24 & 153 \\
\cline { 2 - 3 } & Above: & 73 & \\
& Diagonal: & 308 & \\
& Below: & 69 &
\end{tabular}

\begin{tabular}{rrcc|} 
F2 & $\mathrm{SH}_{k+1}$ & $\mathrm{CP}_{k+1}$ & $\mathrm{OD}_{k+1}$ \\
\cline { 2 - 4 } $\mathrm{SH}_{k}$ & 276 & 2 & 8 \\
$\mathrm{CP}_{k}$ & 3 & 87 & 9 \\
$\mathrm{OD}_{k}{ }_{k}$ & 5 & 10 & 50 \\
\cline { 2 - 4 } & Above: & 19 & \\
& Diagonal: & 413 & \\
& Below: & 18 &
\end{tabular}

\begin{tabular}{r|ccc|}
$\mathrm{F} 3$ & $\mathrm{SH}_{k+1}$ & $\mathrm{CP}_{k+1}$ & $\mathrm{OD}_{k+1}$ \\
\cline { 2 - 4 } $\mathrm{SH}_{k}$ & 293 & 8 & 8 \\
$\mathrm{CP}_{k}$ & 5 & 69 & 9 \\
$\mathrm{OD}_{k}$ & 9 & 10 & 39 \\
\cline { 2 - 4 } & Above: & 25 & \\
& Diagonal: & 401 & \\
& Below: & 24 &
\end{tabular}

\begin{tabular}{r|ccc|}
$\mathrm{F} 4$ & $\mathrm{SH}_{k+1}$ & $\mathrm{CP}_{k+1}$ & $\mathrm{OD}_{k+1}$ \\
\cline { 2 - 4 } $\mathrm{SH}_{k}$ & 432 & 0 & 0 \\
$\mathrm{CP}_{k}$ & 0 & 13 & 2 \\
$\mathrm{OD}_{k}{ }_{k}$ & 0 & 2 & 1 \\
\cline { 2 - 3 } & Above: & 2 & \\
& Diagonal: & 446 & \\
Below: & 2 &
\end{tabular}

\begin{tabular}{r|ccc|} 
F5 & $\mathrm{SH}_{k+1}$ & $\mathrm{CP}_{k+1}$ & $\mathrm{OD}_{k+1}$ \\
\cline { 2 - 4 } $\mathrm{SH}_{k}$ & 392 & 4 & 4 \\
$\mathrm{CP}_{k}$ & 4 & 5 & 6 \\
$\mathrm{OD}_{k}$ & 6 & 3 & 26 \\
\cline { 2 - 4 } & Above: & 14 & \\
& Diagonal: & 423 & \\
& Below: & 13 &
\end{tabular}

\begin{tabular}{r|ccc|} 
F6 & $\mathrm{SH}_{k+1}$ & $\mathrm{CP}_{k+1}$ & $\mathrm{OD}_{k+1}$ \\
\cline { 2 - 4 } $\mathrm{SH}_{k}$ & 300 & 10 & 10 \\
$\mathrm{CP}_{k}$ & 7 & 20 & 14 \\
$\mathrm{OD}_{k}{ }_{k}$ & 11 & 7 & 71 \\
\cline { 2 - 4 } & Above: & 34 & \\
& Diagonal: & 391 & \\
& Below: & 25 &
\end{tabular}

\begin{tabular}{r|ccc|}
$\mathrm{F} 7$ & $\mathrm{SH}_{k+1}$ & $\mathrm{CP}_{k+1}$ & $\mathrm{OD}_{k+1}$ \\
\cline { 2 - 4 } $\mathrm{SH}_{k}$ & 414 & 3 & 3 \\
$\mathrm{CP}_{k}$ & 2 & 12 & 2 \\
$\mathrm{OD}_{k}$ & 2 & 2 & 10 \\
\cline { 2 - 4 } & Above: & 8 & \\
& Diagonal: & 436 & \\
& Below: & 6 &
\end{tabular}

\begin{tabular}{rrrr} 
F8 & $\mathrm{SH}_{k+1}$ & $\mathrm{CP}_{k+1}$ & $\mathrm{OD}_{k+1}$ \\
\cline { 2 - 4 } $\mathrm{SH}_{k}$ & 408 & 9 & 6 \\
$\mathrm{CP}_{k}$ & 8 & 8 & 2 \\
$\mathrm{OD}_{k}{ }_{k}$ & 6 & 0 & 3 \\
& Above: & 17 & \\
& Diagonal: & 419 & \\
& Below: & 14 &
\end{tabular}

\section{FULL}

(group

\begin{tabular}{rrrr}
$\mathrm{F} 9$ & $\mathrm{SH}_{k+1}$ & $\mathrm{CP}_{k+1}$ & $\mathrm{OD}_{k+1}$ \\
\cline { 2 - 4 } $\mathrm{SH}_{k}$ & 418 & 3 & 2 \\
$\mathrm{CP}_{k}$ & 5 & 17 & 0 \\
$\mathrm{OD}_{k}$ & 4 & 1 & 0 \\
\cline { 2 - 4 } & Above: & 5 & \\
& Diagonal: & 435 & \\
& Below: & 10 &
\end{tabular}


Table 3B. Transition Frequencies by Group: Condition PART

\begin{tabular}{r|ccc|}
$\mathrm{P} 1$ & $\mathrm{SH}_{k+1}$ & $\mathrm{CP}_{k+1}$ & $\mathrm{OD}_{k+1}$ \\
\cline { 2 - 4 } $\mathrm{SH}_{k}$ & 212 & 4 & 12 \\
$\mathrm{CP}_{k}$ & 3 & 114 & 15 \\
$\mathrm{OD}_{k}$ & 9 & 19 & 62 \\
\cline { 2 - 4 } & Above: & 31 & \\
Diagonal: & 388 & \\
Below: & 31 &
\end{tabular}

\begin{tabular}{r|ccc|} 
P2 & $\mathrm{SH}_{k+1}$ & $\mathrm{CP}_{k+1}$ & $\mathrm{OD}_{k+1}$ \\
\cline { 2 - 4 } $\mathrm{SH}_{k}$ & 44 & 2 & 31 \\
$\mathrm{CP}_{k}$ & 1 & 112 & 31 \\
$\mathrm{OD}_{k}{ }_{k}$ & 21 & 28 & 180 \\
\cline { 2 - 3 } & Above: & 64 & \\
Diagonal: & 336 & \\
Below: & 50 &
\end{tabular}

\begin{tabular}{r|ccc|}
$\mathrm{P} 3$ & $\mathrm{SH}_{k+1}$ & $\mathrm{CP}_{k+1}$ & $\mathrm{OD}_{k+1}$ \\
\cline { 2 - 4 } $\mathrm{SH}_{k}$ & 32 & 4 & 33 \\
$\mathrm{CP}_{k}$ & 4 & 38 & 19 \\
$\mathrm{OD}_{k}$ & 24 & 12 & 284 \\
\cline { 2 - 3 } & Above: & 56 & \\
Diagonal: & 354 & \\
Below: & 40 &
\end{tabular}

\begin{tabular}{r|ccc|} 
P4 & $\mathrm{SH}_{k+1}$ & $\mathrm{CP}_{k+1}$ & $\mathrm{OD}_{k+1}$ \\
\cline { 2 - 4 } $\mathrm{SH}_{k}$ & 407 & 0 & 9 \\
$\mathrm{CP}_{k}$ & 1 & 0 & 0 \\
$\mathrm{OD}_{k}$ & 8 & 1 & 24 \\
\cline { 2 - 4 } & Above: & 9 & \\
Diagonal: & 431 & \\
Below: & 10 &
\end{tabular}

\begin{tabular}{r|ccc|} 
P5 & $\mathrm{SH}_{k+1}$ & $\mathrm{CP}_{k+1}$ & $\mathrm{OD}_{k+1}$ \\
\cline { 2 - 4 } $\mathrm{SH}_{k}$ & 8 & 2 & 9 \\
$\mathrm{CP}_{k}$ & 2 & 32 & 20 \\
$\mathrm{OD}_{k}{ }_{k}$ & 5 & 15 & 357 \\
\cline { 2 - 3 } & Above: & 31 & \\
Diagonal: & 397 & \\
Below: & 22 &
\end{tabular}

\begin{tabular}{rrcc|} 
P6 & $\mathrm{SH}_{k+1}$ & $\mathrm{CP}_{k+1}$ & $\mathrm{OD}_{k+1}$ \\
\cline { 2 - 4 } $\mathrm{SH}_{k}$ & 342 & 8 & 7 \\
$\mathrm{CP}_{k}$ & 6 & 64 & 6 \\
$\mathrm{OD}_{k}$ & 5 & 6 & 6 \\
\cline { 2 - 4 } & Above: & 21 & \\
Diagonal: & 412 & \\
Below: & 17 &
\end{tabular}

\begin{tabular}{r|ccc|}
$\mathrm{P} 7$ & $\mathrm{SH}_{k+1}$ & $\mathrm{CP}_{k+1}$ & $\mathrm{OD}_{k+1}$ \\
\cline { 2 - 4 } $\mathrm{SH}_{k}$ & 14 & 0 & 36 \\
$\mathrm{CP}_{k}$ & 2 & 1 & 3 \\
$\mathrm{OD}_{k}$ & 32 & 3 & 359 \\
\cline { 2 - 3 } & Above: & 39 & \\
Diagonal: & 374 & \\
Below: & 37 &
\end{tabular}

\begin{tabular}{rrcc|} 
P8 & $\mathrm{SH}_{k+1}$ & $\mathrm{CP}_{k+1}$ & $\mathrm{OD}_{k+1}$ \\
\cline { 2 - 4 } $\mathrm{SH}_{k}$ & 325 & 0 & 12 \\
$\mathrm{CP}_{k}$ & 3 & 1 & 0 \\
$\mathrm{OD}_{k}$ & 9 & 3 & 97 \\
\cline { 2 - 4 } & Above: & 12 & \\
Diagonal: & 423 & \\
Below: & 15 &
\end{tabular}

PART

\begin{tabular}{r|ccc} 
P9 & $\mathrm{SH}_{k+1}$ & $\mathrm{CP}_{k+1}$ & $\mathrm{OD}_{k+1}$ \\
\cline { 2 - 4 } $\mathrm{SH}_{k}$ & 363 & 9 & 3 \\
$\mathrm{CP}_{k}$ & 10 & 58 & 1 \\
$\mathrm{OD}_{k}$ & 2 & 2 & 2 \\
\cline { 2 - 3 } & Above: & 13 & \\
Diagonal: & 423 & \\
Below: & 14 &
\end{tabular}

\begin{tabular}{rccc|}
\multicolumn{1}{c}{ (group } & & & \\
average) & $\mathrm{SH}_{k+1}$ & $\mathrm{CP}_{k+1}$ & $\mathrm{OD}_{k+1}$ \\
\cline { 2 - 4 } $\mathrm{SH}_{k}$ & $\mathbf{1 9 4}$ & 3 & 17 \\
$\mathrm{CP}_{k}$ & 4 & $\mathbf{4 7}$ & 11 \\
$\mathrm{OD}_{k}$ & 13 & 10 & $\mathbf{1 5 2}$ \\
\cline { 2 - 4 } & Above: & 31 & \\
Diagonal: & 393 & \\
Below: & 26 &
\end{tabular}


Table 4. Individual-Level Measures by Condition (s.d. in parentheses)

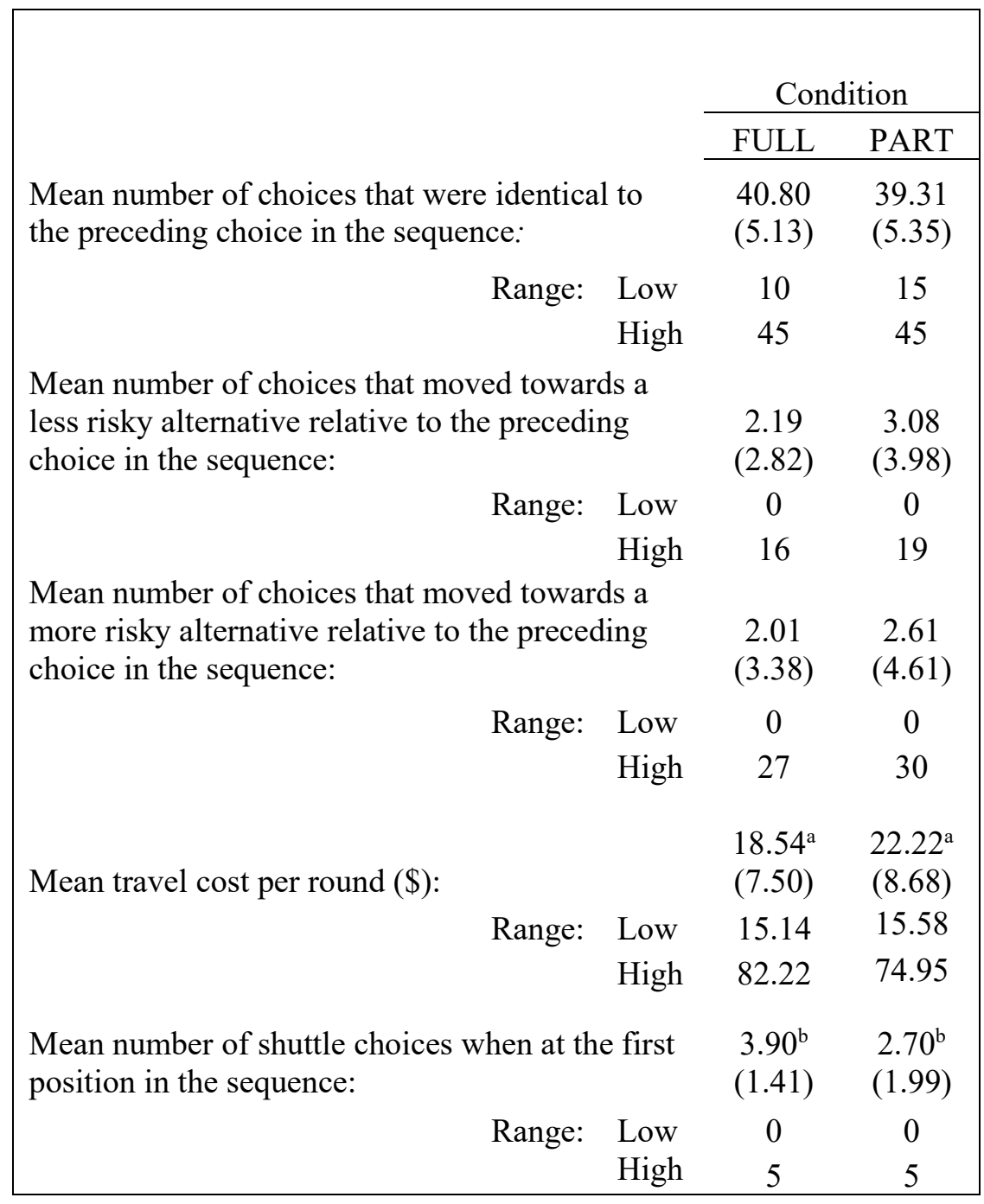

${ }^{\mathrm{a}} t(178)=3.05, p<0.01 \quad{ }^{\mathrm{b}} t(178)=4.66, p<0.001$ 


\section{Appendix: Subject Instructions}

Welcome to an experiment on cost sharing. During the present experimental session, you will be asked to make a large number of decisions and so will the other participants. Your decisions, as well as the decisions of the other participants, will determine your monetary payoff according to a procedure that will be explained below.

Please read the instructions carefully. If at any time during the session you have questions, please raise your hand and one of the experimenters will come to assist you. You may refer to the instructions during any time in the session.

Please note that from now on all communication between the participants is prohibited. If the participants communicate with one another in any shape or form, the session will be terminated. Please note, too, that the experiment is self-paced. Therefore, you may anticipate short delays while other participants in your group determine and then type in their decisions.

\section{Description of the Task}

Consider yourself to be one of $\mathbf{1 0}$ commuters, each owning a car. In this experiment, you will be asked to participate in a task that simulates the decisions commuters often face in choosing among alternative modes of transportation between a given origin (say, a convention center) and a given destination (say, a restaurant for the conference dinner). The game consists of many identical rounds. Each round is described as follows.

The commuters (hereafter called "players") will be asked to make their decisions one by one following an order which is pre-determined randomly by the computer. Each player has to choose one of three alternatives:

- $\mathbf{O} \rightarrow \mathbf{D}$ : Drive your car by yourself from the origin (denoted by the letter $\mathbf{O}$ ) to the destination (denoted by the letter $\mathbf{D}$ ). The cost for traveling by yourself in your own car is $\$ \mathbf{2 0 . 0 0}$

- $\mathbf{O} \rightarrow \mathbf{C P} \rightarrow \mathbf{D}:$ Drive your car from the origin to a nearby carpool lot, park your car, and then travel with the carpool to your destination. You pay $\$ \mathbf{1 0 . 0 0}$ for parking your car. In addition, the cost charged by the carpool is $\$ \mathbf{7 0 . 0 0}$, which is divided equally by the number of players who 
choose the carpool. The capacity of the carpool is 10. In other words, you share equally the cost of public transportation. Thus, your travel cost from $\mathbf{O}$ to $\mathbf{D}$ through route $\mathbf{O} \rightarrow \mathbf{C P} \rightarrow \mathbf{D}$ is

$$
\$ 10+\frac{\$ 70}{\mathrm{~m}(\mathrm{CP} \rightarrow \mathrm{D})},
$$

where $\mathbf{m}(\mathbf{C P} \rightarrow \mathbf{D})$ is the number of players who share the carpool.

- $\mathbf{O} \rightarrow \mathbf{S H} \rightarrow \mathbf{D}$ : Drive your car from the origin to a shuttle station, park your car, and travel with the shuttle to your destination. There is no charge for parking your car in the shuttle station. The total cost of the shuttle is $\mathbf{\$ 1 5 0 . 0 0}$, which is divided equally by the number of players riding the shuttle. The capacity of the shuttle is 10 . Thus, your travel cost from $\mathbf{O}$ to $\mathbf{D}$ through route $\mathbf{O} \rightarrow \mathbf{S H} \rightarrow \mathbf{D}$ is

$$
\frac{\$ 150}{\mathrm{~m}(\mathrm{SH} \rightarrow \mathrm{D})},
$$

where $\mathbf{m}(\mathbf{S H} \rightarrow \mathbf{D})$ is the number of players who share the ride by the shuttle.

This is a sequential game in which players make their decisions one by one. Each player will be assigned a different position in the sequence (from 1 to 10 ) at the beginning of each round. Then, each player will be asked to choose one of the three alternatives when it is her turn to play. Moreover, before confirming her choice, she will be informed of the choice of the last player who preceded her in the sequence. The game will be iterated for 50 rounds; they only differ from each other in the assignment of the positions.

\section{Experimental Procedure}

At the beginning of each round, each of the ten players will be presented with the following diagram. A message window will pop up to indicate the round number and your position in the sequence of choosing the mode of transportation. The position of each player in the sequence will randomly be assigned by the computer, and in general will change from one round to another. During the experiment, each player will be assigned any given position the same number of times. 


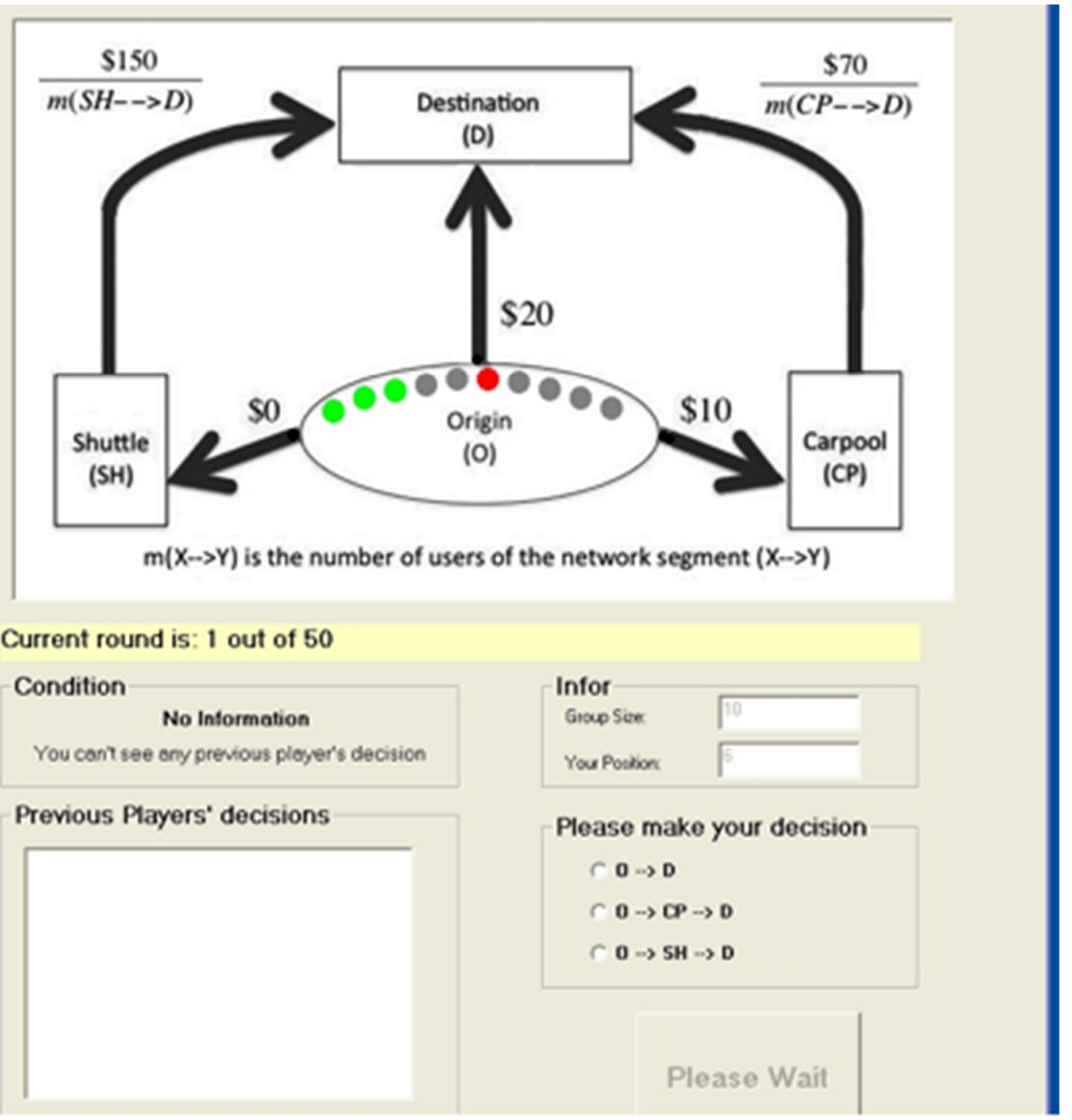

Please study the main screen above. The upper part of the screen is a sketch map of the traffic system. There are 10 cars parked in the origin; each is exhibited as a solid circle. When it is her turn to play, each player can either drive to the destination $\mathbf{D}$ (up arrow) and pay $\$ 20.00$, drive to the carpool station (right arrow), park her car, and then take the carpool for a total cost of $\$ 10+\$ 70 / \mathbf{m}(\mathbf{C P} \rightarrow \mathbf{D})$, or drive to the shuttle station (left arrow), park her car (for free), and then take the shuttle for a total cost of $\$ 150 / \mathbf{m}(\mathbf{S H} \rightarrow \mathbf{D})$.

On the screen, grey solid circles represent players who are waiting to be called to make their decision. Green solid circles represent players who have already made their decisions. And the single red solid circle represents each player's current position. After each player makes her 
decision, her red solid circle will turn to green. The middle of the screen shows a status bar (in yellow) that indicates the progress of the experiment. It tells you how many rounds have been completed and the total number of rounds.

In the lower part of the screen there are two tables. The top table on the right, labeled infor, shows the group size (which is 10) and your position in the sequence. The bottom table is where you make your decision. Use the mouse to choose one of the three options. If you change your mind, please choose another option. Your decision will not register until you press the Confirm button (lower right). The left part of the lower screen, labeled Condition, tells you if you have any information about the decisions of the other players. Here it says Partial Information, meaning that the decision of the last player who preceded you in the sequence is displayed.

Once all the ten players type in their decisions and confirm them, a new screen will pop up to display the outcome of the round. This screen shows the number of players who have chosen each of three routes, the cost associated with each decision, your decision, your cost for the round, and your payoff in points. See an example in screen below.

\section{Please study the outcome screen below.}

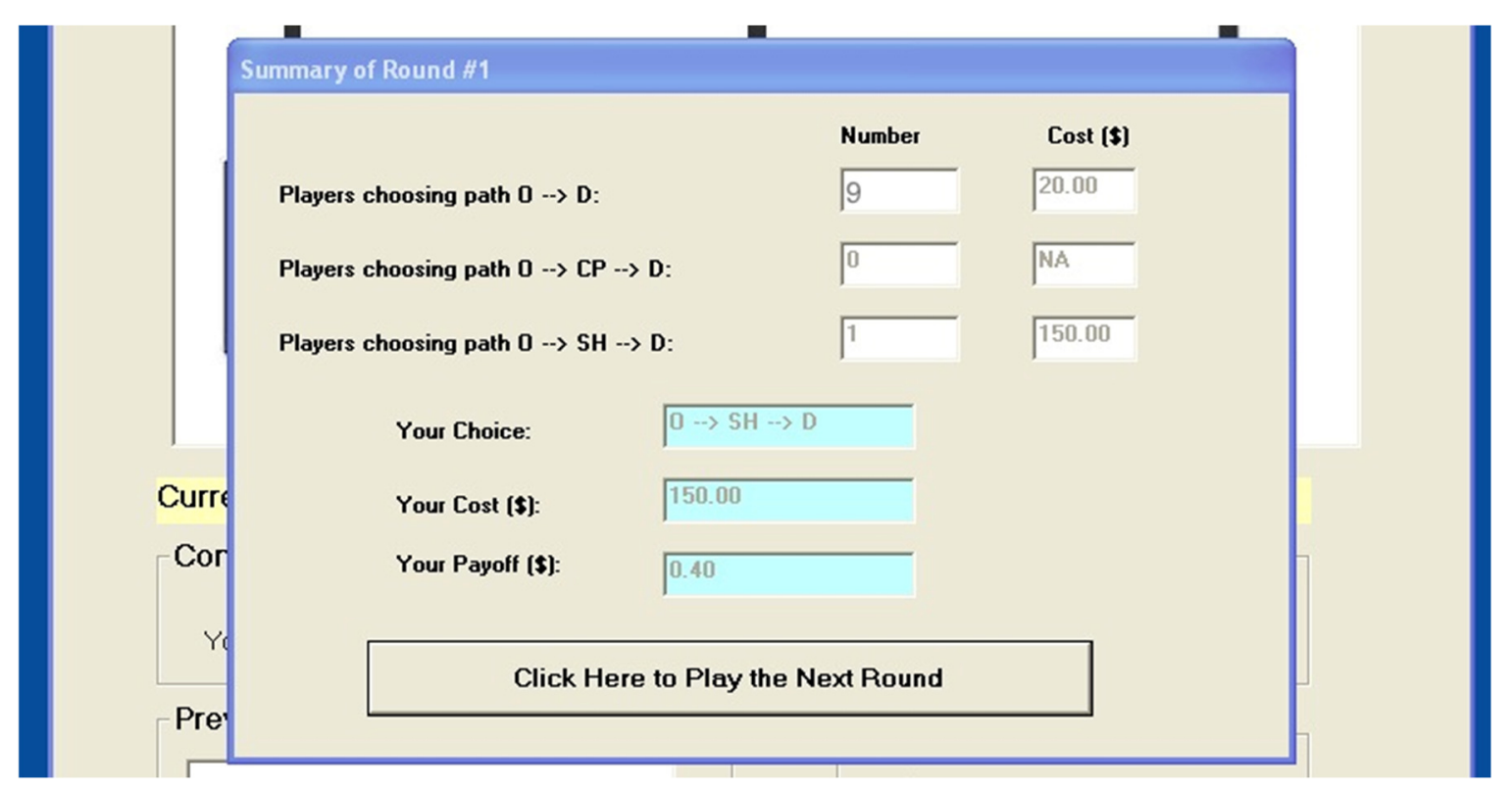


After clicking the "Click here to play the next round" at the bottom of this screen, a window will pop up instructing the player to wait for all the players to review the outcome of the round. Then, a new round will start. The game will be repeated in this way for 50 rounds. The only difference from one round to another is in the order of the players that are assigned randomly by the computer.

How will you be paid?

When all the 50 rounds have been completed, the computer will choose randomly 5 payoff rounds for each player. Payoff of each round will then be converted into US dollars at the rate:

$$
\text { Payoff }=\frac{\$ 90}{\text { cost }}
$$

For example, if your cost in a chosen round is $\$ 20$, your payoff in that round will be $\$(90 / 20)=$ $\$ 4.5$. Your final payoff will be the sum of the payoffs from the 5 randomly selected rounds. In addition, you will be paid $\$ 5.00$ for your participation in the experiment. The experimenter will come up to your cubicle and write your total payoff on your receipt. You'll complete the receipt, sign it up, and then be paid cash by one of the experimenters.

Please place the instructions on the table in front of you to indicate that you have completed reading them. The experiment will begin shortly. Please remember that no communication is allowed during the experiment. If you encounter any difficulties please raise your hand and you will be responded to by the experimenter.

Thank you! 